\title{
A NOTE ON THE SCHRÖDINGER OPERATOR WITH EXPONENTIAL POTENTIAL
}

\author{
AGIL KH. KHANMAMEDOV AND AFAG F. MAMEDOVA
}

\begin{abstract}
The Schrödinger operator $L=-\frac{d^{2}}{d x^{2}}+e^{2 x}$ on the positive half-line with the Dirichlet boundary condition at zero is studied. A description of the domain of definition of the operator $L$ is given. Using the connection between the modified Bessel equation and the Schrödinger equation, the distribution of the zeros of the Macdonald function and its derivative is investigated.
\end{abstract}

\section{Introduction and main result}

Quantum mechanics gave a powerful impetus to the development of the spectral theory of differential operators with increasing coefficients. In particular, various spectral problems for the Schrödinger operator with increasing potential were studied very extensively (see [2], [4], [7], [8], [10], [13]). Some problems of conformal field theory are closely related to the Schrödinger operator with an exponential potential (see [14]). However, the question of describing the domain of definition of such operators has not been studied previously.

We consider the operator $L$ defined on the space $L_{2}(0,+\infty)$ by the differential expression

$$
l(y)=-y^{\prime \prime}+e^{2 x} y, x \in[0,+\infty)
$$

with the domain

$$
D(L)=\left\{y \in L_{2}(0,+\infty): y \in W_{2, l o c}^{2}, l(y) \in L_{2}(0,+\infty), y(0)=0\right\} .
$$

In this paper we describe the domain of definition of the operator $L$. It should be noted that the last problem is closely related to the modified Bessel equation

$$
z^{2} u^{\prime \prime}+z u^{\prime}-\left(z^{2}+\nu^{2}\right) u=0 .
$$

The study of the zeros of solutions to the equation (1.1) is of independent interest (e.g., see [3], [6], [9], [11],[12]).

Consider the equation (1.1) for $z>0$. If we set $z=e^{x+c}, y(x)=u\left(e^{x+c}\right), \nu=$ $i \lambda$, equation (1.1) takes the form

$$
-y^{\prime \prime}+e^{2(x+c)} y=\lambda^{2} y
$$

2010 Mathematics Subject Classification. 34A55, 34L40.

Key words and phrases. Schrödinger operator, increasing potential, eigenvalues, Bessel functions, zeros of Bessel functions. 
In what follows, we deal with special functions satisfying the equation (1.2). It is well known (see [1], [14]) that this equation has two linearly independent solutions $\varphi(x, \lambda)=I_{i \lambda}\left(e^{x+c}\right)$ and $\psi(x, \lambda)=K_{i \lambda}\left(e^{x+c}\right)$, where $I_{\nu}(z)$ and $K_{\nu}(z)$ are modified functions of the first and second kind, respectively. For each $z>0$ both these modified Bessel functions are entire functions of the index $\nu$. The function $K_{\nu}(z)$ satisfies [1] the representation

$$
K_{i \lambda}(z)=\int_{0}^{\infty} e^{-z c h t} \cos \lambda t d t,|\arg z|<\frac{\pi}{2}, \lambda \in C .
$$

In particular, this implies that $K_{0}(z)>0, z>0$. We need some (see [1]) asymptotic equalities related to the functions $I_{\nu}(z)$ and $K_{\nu}(z)$

$$
\begin{gathered}
I_{\nu}(z)=\frac{e^{z}}{\sqrt{2 \pi z}}\left(1+O\left(\frac{1}{z}\right)\right), z \rightarrow \infty,|\arg z|<\frac{\pi}{2}, \\
I_{\nu}^{\prime}(z)=\frac{e^{z}}{\sqrt{2 \pi z}}\left(1+O\left(\frac{1}{z}\right)\right), z \rightarrow \infty,|\arg z|<\frac{\pi}{2}, \\
K_{\nu}(z)=\sqrt{\frac{\pi}{2 z}} e^{-z}\left(1+O\left(\frac{1}{z}\right)\right), z \rightarrow \infty,|\arg z|<\frac{3 \pi}{2}, \\
K_{\nu}^{\prime}(z)=-\sqrt{\frac{\pi}{2 z}} e^{-z}\left(1+O\left(\frac{1}{z}\right)\right), z \rightarrow \infty,|\arg z|<\frac{3 \pi}{2} .
\end{gathered}
$$

We also introduce the special solutions

$$
\begin{gathered}
\psi(x)=K_{0}\left(e^{x}\right), \\
\varphi(x)=I_{0}\left(e^{x}\right)-\frac{I_{0}(1)}{K_{0}(1)} K_{0}\left(e^{x}\right)
\end{gathered}
$$

of the equation (1.2) with $\lambda=0, c=0$.

The main result of this paper is the following theorem.

Theorem 1.1. The domain $D(L)$ coincides with the set of functions of the form

$$
y(x)=\psi(x) \int_{0}^{x} \varphi(t) f(t) d t+\varphi(x) \int_{x}^{+\infty} \psi(t) f(t) d t,
$$

where $f(x)$ ranges over the entire space $L_{2}(0,+\infty)$. For each function $y(x) \in$ $D(L)$, one has

$$
e^{x} y(x) \rightarrow 0, y^{\prime}(x) \rightarrow 0, e^{x} y(x), y^{\prime}(x) \in L_{2}(0,+\infty)
$$

Equation (1.9) defines a bounded operator on $L_{2}(0,+\infty)$, which is the inverse of $L$.

\section{Proof of the theorem}

Obviously, the operator $L$ is densely defined, because its domain contains infinitely differentiable functions compactly supported on $(0, \infty)$; the set of these functions is well known to be dense in $L_{2}(0, \infty)$. Moreover, the operator $L$ is selfadjoint. Further, note that the $\psi(x)=K_{0}\left(e^{x}\right)$ decays like a double exponent as $x \rightarrow \infty$. Hence the improper integral in (1.9) converges. Since $f(x) \in L_{2}(0,+\infty)$, 
it follows that the function $y=y(x)$ defined in (1.9) lies in $W_{2}^{1}[0, b]$ for every finite $b$. By differentiating, we obtain

$$
y^{\prime}(x)=\psi^{\prime}(x) \int_{0}^{x} \varphi(t) f(t) d t+\varphi^{\prime}(x) \int_{x}^{+\infty} \psi(t) f(t) d t,
$$

whence it follows that $y^{\prime}(x) \in W_{2}^{1}[0, b]$ for every finite $b$. By differentiating once more, we obtain

$$
\begin{aligned}
& y^{\prime \prime}(x)=\left[\psi^{\prime}(x) \varphi(x)-\psi(x) \varphi^{\prime}(x)\right] f(x)+\psi^{\prime \prime}(x) \int_{0}^{x} \varphi(t) f(t) d t+ \\
& +\varphi^{\prime \prime}(x) \int_{x}^{+\infty} \psi(t) f(t) d t=-f(x)+e^{2 x} y(x),
\end{aligned}
$$

i.e., $y(x) \in W_{2}^{2}[0, b]$ for each $b>0$ and $\ell(y)=f(x) \in L_{2}(0,+\infty)$. Since $y(0)=0$, it follows that $y(x) \in D(L)$. The converse is true as well. Namely, let $y \in D(L)$ and $\ell(y)=f(x) \in L_{2}(0,+\infty)$. By a classical theorem on the general form of a solution of a differential equation,

$$
y(x)=C_{1} \psi(x)+C_{2} \varphi(x)+\psi(x) \int_{0}^{x} \varphi(t) f(t) d t+\varphi(x) \int_{x}^{+\infty} \psi(t) f(t) d t,
$$

where $C_{1}$ and $C_{2}$ are constants. It follows from the relation $y(0)=0$ that $C_{1}=0$, while the condition $y \in L_{2}(0,+\infty)$ and the estimate $(2.3)$, which will be proved below, imply that $C_{2}=0$; i.e., $y$ admits the representation (1.9). Thus, formula (1.9) defines the inverse operator $L^{-1}$. Its boundedness follows from the estimate $|y(x)| \leq R\|f\|$ on every finite interval $[0, b]$ and the estimate (2.3). Here and in what follows, the letter $R, R_{j}, j=1,2,3$ stands for various positive constants, and $\|\circ\|=\|\circ\|_{L_{2}(0, \infty)}$.

Let us prove relations (1.10). First, note that, by virtue of (1.3), (1.8), there exists a constant $R$ such that the estimate

$$
|\varphi(t)| \leq R e^{-\frac{t}{2}} e^{e^{t}}
$$

holds for $t>0$. It is easily seen that the function $g(t)=e^{-\frac{t}{2}} e^{e^{t}}$ increases for sufficiently large $t>b$. Hence

$$
\begin{aligned}
& \left|\int_{0}^{x} \varphi(t) f(t) d t\right| \leq\left(\int_{0}^{b}+\int_{b}^{x-1}+\int_{x-1}^{x}\right)|\varphi(t)||f(t)| \leq R_{1}\|f\|+ \\
& +R x^{\frac{1}{2}} e^{-\frac{x-1}{2}} e^{e^{x-1}}\|f\|+R e^{-\frac{x}{2}} e^{e^{x}}\left(\int_{x-1}^{x}|f(t)|^{2} d t\right)^{\frac{1}{2}}
\end{aligned}
$$

for $x>b+1$. Here the constant $R_{1}$ depends on $b$ alone. This estimate, together with the representation (1.5), implies that

$$
\begin{aligned}
& \left|\psi(x) \int_{0}^{x} \varphi(t) f(t) d t\right| \leq R_{2}\|f\| e^{-\frac{x}{2}} e^{-e^{x}}+ \\
& +R_{2} x^{\frac{1}{2}} e^{-x} e^{e^{x}\left(e^{-1}-1\right)}\|f\|+R_{2} e^{-x}\left(\int_{x-1}^{x}|f(t)|^{2} d t\right)^{\frac{1}{2}}
\end{aligned}
$$

for sufficiently large $x$. We have estimated the first summand in (1.9). In a similar way, we estimate the second summand. The function $h(t)=e^{t} e^{-e^{t}}$ is decreasing for sufficiently large $t$, and due to (1.5), (1.7) we have

$$
\begin{aligned}
& \left|\int_{x}^{\infty} \psi(t) f(t) d t\right| \leq\left(\int_{x}^{x+1}+\int_{x+1}^{\infty}\right)|\psi(t)||f(t)| \leq \\
& \leq R \int_{x}^{x+1} h(t) e^{-\frac{3}{2} t}|f(t)| d t+R\left(\int_{x+1}^{\infty} h^{2}(t) e^{-3 t} d t\right)^{\frac{1}{2}}\|f\| \leq \\
& \leq R h(x) e^{-\frac{3}{2} x}\left(\int_{x}^{x+1}|f(t)|^{2} d t\right)^{\frac{1}{2}}+R h(x+1) e^{-x}\|f\|
\end{aligned}
$$


for large $x$. We have

$$
|\varphi(x)| \leq R e^{\frac{x}{2}} h^{-1}(x), h(x+1) h^{-1}(x)=e e^{(1-e) e^{x}}
$$

and hence the absolute value of the second term on the right-hand side in (1.9) can be estimated by

$$
R e^{-x}\left(\int_{x}^{x+1}|f(t)|^{2} d t\right)+R e e^{-\frac{x}{2}} e^{(1-e) e^{x}}\|f\| .
$$

By adding the resulting estimates, we arrive at the inequality

$$
\begin{aligned}
& |y(x)| \leq R_{3} x^{\frac{1}{2}} e^{-x} e^{(1-e) e^{x}}\|f\|+R_{3} e^{-\frac{x}{2}} e^{(1-e) e^{x}}\|f\|+ \\
& R_{3} e^{-x}\left(\int_{x-1}^{x+1}|f(t)|^{2} d t\right)^{\frac{1}{2}}, x>b+1
\end{aligned}
$$

which proves the first relation in (1.10). The second relation in (1.10) can be obtained in the same way except that (2.2) is used instead of (1.9) and we take into account the fact that the estimates for the derivatives $\psi(x)$ and $\varphi(x)$ differ from the estimates for the functions themselves by the factor $e^{x}$ (see (1.4), (1.6)$(1.8))$.

Let us prove that $e^{x} y(x) \in L_{2}(0,+\infty)$. It follows from the estimate $(2.2)$ that

$$
\begin{aligned}
& \int_{b}^{\infty}\left|e^{x} y(x)\right|^{2} d x \leq R\|f\|^{2} \int_{b}^{\infty} x e^{2(1-e) e^{x}} d x+R\|f\|^{2} \int_{b}^{\infty} e^{x} e^{2(1-e) e^{x}} d x+ \\
& +R \int_{b}^{\infty} \int_{x-1}^{x+1}|f(t)|^{2} d t d x \leq R\|f\|^{2}+R \int_{b-1}^{\infty}|f(t)|^{2} \int_{t-1}^{t+1} d x d t \leq R\|f\|^{2} .
\end{aligned}
$$

The inclusion $y^{\prime}(x) \in L_{2}(0,+\infty)$ can be obtained in a similar way with regard to the fact that $|y(x)|$ is bounded by the right-hand side of $(2.2)$ multiplied by $e^{x}$. This completes the proof of the theorem.

The above results also extend to the case of the self-adjoint operator $L_{c}$, generated on the space $L_{2}(0,+\infty)$ by the differential expression

$$
l_{c}(y)=-y^{\prime \prime}+e^{2(x+c)} y, x \in[0,+\infty)
$$

and boundary condition

$$
\alpha y(0)+\beta y^{\prime}(0)=0,
$$

where $\alpha^{2}+\beta^{2}>0$. By way of application, we use the operator $L_{c}$. Since $e^{x} \rightarrow+\infty$ as $x \rightarrow+\infty$, the spectrum of the operator $L_{c}$ consists [4] of simple real eigenvalues $\lambda_{n}^{2}=\lambda_{n}^{2}(c)>0, n=1,2, \ldots$, condensing to $+\infty$. Hence and from the relationship $K_{\nu}(z)=K_{-\nu}(z)$ it follows that the function

$$
\Delta(\lambda)=\alpha K_{i \lambda}\left(e^{c}\right)+\beta e^{c} K_{i \lambda}^{\prime}\left(e^{c}\right)
$$

has only real zeros $\pm \lambda_{n}, \lambda_{n}>0, n=1,2, \ldots$ Further, in the boundary condition (2.4) the quantities $\alpha, \beta$ can be arbitrary numbers that satisfy the condition $\alpha^{2}+\beta^{2}>0$. Obviously, numbers $a=\alpha, b=\beta e^{c}$ also have this property. Assuming then $z=e^{c}$ and taking into account that $\nu=i \lambda$ from (2.5) we find that the function $a K_{\nu}(z)+b K_{\nu}^{\prime}(z), z>0$ has a countable number of simple purely imaginary zeros $\pm i \nu_{n}, \nu_{n}>0, n=1,2, \ldots$. 


\section{References}

[1] M. Abramowitz and I. A. Stegun, eds., Handbook of Mathematical Functions with Formulas, Graphs, and Mathematical Tables (Natl. Bur. Stds. Appl. Math. Ser., Vol. 55), Dover, New York (1972).

[2] S. M. Bagirova and A. Kh. Khanmamedov, The inverse spectral problem for the perturbed harmonic oscillator on the entire axis, Proc. Inst. Math. Mech. Natl. Acad. Sci. Azerb., 44(2018), 285-294.

[3] S. M. Bagirova and A. Kh. Khanmamedov, On Zeros of the Modified Bessel Function of the Second Kind, Computational Mathematics and Mathematical Physics, 60(205) (2020), 817-820.

[4] F. A. Berezin and M. A. Shubin, The Schrödinger Equation, Kluwer, Dordrecht (1991).

[5] F. Calogero and A. Degasperis, Inverse spectral problem for the one- dimensional Schrödinger equation with an additional linear potential, Lett. Nuovo Cimento, 23 (1978), 143-149.

[6] M. J. Coulomb, Sur les zéros des fonctions de Bessel considérées comme fonctions de 1 ordre. Bull. Sci. Math., 60(1936), 297-302.

[7] M. G. Gasymov and B. A. Mustafaev, On the inverse problem of scattering theory for the anharmonic equationon a semiaxis, Soviet Math. Dokl., 17(1976), 621-624.

[8] I. M. Guseinov, A. Kh. Khanmamedov, and A. F. Mamedova, Inverse scattering problem for the Schrödinger equation with an additional quadratic potential on the entire axis, Theor. Math. Phys., 195(2018), 538-547.

[9] G. D.J. Hejhal, On a result of G. Polya concerning the Riemann function, J. Anal. Math., 55 (1990), 59-95.

[10] Y. Li, One special inverse problem of the second order differential equation on the whole real axis, Chinese Ann. Math., 2(1981), 147-155.

[11] G.Polya, On the zeros of certain trigonometric integrals, J. of London Math. Soc., 1(2) (1926), 98-99.

[12] G. Polya, Bemerkung uber die lntegraldarstellung der Riemannschen Funktion, Acta Math. 48 (1926), 305-317.

[13] A. M. Savchuk and A. A. Shkalikov, Spectral Properties of the Complex Airy Operator on the Half-Line. Functional Analysis and Its Applications, 51(1) (2017), 66-79.

[14] L. A. Takhtajan and L. D. Faddeev, The spectral theory of a functional-difference operator in conformal field theory. Izvestiya: Mathematics, 79(2) (2015),388-411.

Agil Kh. Khanmamedov

Institute of Mathematics and Mechanics, National Academy of Sciences of Azerbaijan, B. Vahabzadeh 9, Baku, AZ1141, Azerbaijan;

Baku State University, Z. Khalilov 23, Baku, AZ1148, Azerbaijan

E-mail address: agil_khanmamedov@yahoo.com

Afag F. Mamedova

Azerbaijan State University of Economics (UNEC), AZ 1001, Baku, Azerbaijan

E-mail address: mammadova_afaq@unec.edu.az

Received: September 25, 2020; Accepted: February 4, 2021 